\title{
DESEMPENHO DE CORDEIROS NATURALMENTE INFECTADOS COM PARASITAS GASTRINTESTINAIS UTILIZANDO O TRATAMENTO SELETIVO COM O MÉTODO FAMACHA E O TRATAMENTO PREVENTIVO
}

\author{
(Growth performance in naturally infected lambs under selective treatment \\ with FAMACHA method and preventive treatment)
}

\author{
DEPNER, R. A. ${ }^{1}$; GAVIÃO, A. A. ${ }^{1}$; CECIM, M. ${ }^{1}$; ROCHA, R. ${ }^{1}$; MOLENTO, M. B. ${ }^{2}$
}

1 Centro de Ciências Rurais, Universidade Federal de Santa Maria, UFSM. 2 Universidade Federal do Paraná, UFPR. R: dos Funcionários, 1540. Curitiba, PR CEP: 80035-050. E-mail: molento@ufpr.br

\begin{abstract}
RESUMO - O objetivo do presente trabalho foi avaliar o ganho de peso em cordeiros utilizando duas práticas de controle parasitário entre 14/11/04 a 14/03/05. Trinta e quatro animais da raça lle de France compuseram dois grupos de 17 animais (G1 e G2). O grupo G1 foi avaliado em intervalos de 14 dias utilizado o método Famacha, o escore de diarréia, exame de fezes (OPG) e o escore de condição corporal na mesma freqüência, sendo tratados com moxidectina caso apresentassem comprometimento clínico. O grupo $\mathrm{G} 2$ foi tratado com o mesmo princípio ativo em intervalos de 30 dias. A correlação de valores Famacha entre 2, 3 e 4 e as variáveis OPG e peso foi de 0,903 e 0,662 , respectivamente. Os grupos não apresentaram diferença no ganho de peso total $(P=0,110)$. Houve predominância da espécie Haemonchus contortus para G1 $(62,94 \%)$ e G2 (64,14\%). A opção de tratar os animais com o sistema seletivo reduziu em $87,5 \%$ o custo do tratamento, quando comparado com o regime preventivo. O tratamento seletivo com o método Famacha permite um controle parasitário eficiente quando existe alta prevalência do parasita $H$. contortus. Estes dados permitem inferir sobre a possibilidade de seleção de animais menos tolerantes durante um período de maior desafio parasitário.
\end{abstract}

Palavras-chave: cordeiros; Haemonchus contortus; tratamento seletivo; tratamento preventivo; Famacha.

\begin{abstract}
The objective of the present work was to evaluate the weight gain in lambs using two systems of parasite control in between 14/11/04 and $14 / 03 / 05$. Thirty four animals of the lle de France breed where used in two groups of 17 animals (G1 and G2). The G1 group was evaluated at 14-days interval using Famacha, diarrhea and body condition score and fecal exam (EPG) at the same frequency being treated with moxidectin in case they manifested any clinical sign. The $\mathrm{G} 2$ group was treated with the same compound at 30-days interval. The correlation of Famacha 2, 3 and 4 and the variables EPG and weight was of 0,903 and 0,662 , respectively. The groups demonstrated no difference on their final weight gain $(P=0,110)$. There was the predominance of the Haemonchus contortus species for G1 (62,94\%) and G2 (64,14\%). The option to treat animals using the selective system reduced by $87,5 \%$ the treatment cost when compared with the preventive regime. The target selective treatment using the Famacha method allows a very efficient parasite control when there is a high prevalence of the parasite $H$. contortus. The data permit inferring about the possibility to select less tolerant animals during a period of heavier parasite challenge.
\end{abstract}

Key-words: lambs; Haemonchus contortus; selective treatment; preventive treatment; Famacha.

\footnotetext{
* Laboratório de Doenças Parasitárias, Setor de Ciências Agrárias, Universidade Federal do Paraná, UFPR. R: dos Funcionários, 1540. Curitiba, PR CEP: 80035-050. E-mail: molento@ufpr.br. Fone: (41) 3350-5618, FAX: (41) 3350-5623
} 
Desempenho de cordeiros naturalmente infectados com nematodas gastrintestinais utilizando o tratamento seletivo com o método famacha e o tratamento preventivo

\section{INTRODUÇÃO}

A principal enfermidade em ovinos é causada por parasitas gastrintestinais (KAPLAN et al., 2004). O Haemonchus contortus é o principal parasita de pequenos ruminantes e é, isoladamente, um fator limitante para a cadeia produtiva da ovinocultura e caprinocultura. Este nematóide causa grande retirada de sangue, ocasionando a diminuição da produção e da qualidade da lã e queda de 20 a $40 \%$ no ganho de peso dos animais (KAWANO et al., 2001).

O controle desta enfermidade tem o objetivo de permitir o desempenho zootécnico desejável dos animais e é realizado com a utilização de antihelmínticos em intervalos curtos e com a alternância rápida de bases (MOLENTO e ALMEIDA, 2004). O tratamento preventivo/supressivo promove a seleção de organismos geneticamente e fisiologicamente resistentes, que sobreviverão ao contato com drogas anti-helmínticas (PRICHARD, 1994). A resistência anti-helmíntica múltipla foi determinada em vários países, como Brasil, Paraguai, África do Sul e Malásia (ECHEVARRIA et al., 1996; MACIEL et al., 1996; VAN WYK et al., 1999; CHANDRAWATHANI et al., 2003). Pesquisadores alertam que, com a utilização de drogas de forma pouco criteriosa, muito em breve haverá a redução das fontes de controle químico causando prejuízo para produtores que dependem desta atividade (MOLENTO e PRICHARD, 1999; VAN WYK et al., 1999).

Contudo, existe a necessidade de ratificar métodos de controle parasitário que não dependam completamente do controle químico contra $\mathrm{H}$. contortus, e que possuam vantagens econômicas e zootécnicas, visando a sustentabilidade da produção agropecuária. O método Famacha consiste em avaliar os animais individualmente, comparando diferentes graus da coloração da conjuntiva (VAN WYK et al., 1997) sendo este incluído no Sistema Integrado de Controle Parasitário, SICOPA desenvolvido para as condições do produtor no Brasil (MOLENTO, 2004). BATH e VAN WYK (2001) demonstraram a freqüência com que ovelhas necessitaram receber tratamento durante a estação do verão com o método Famacha e dados de peso médio. Entre 250 animais, 32,4; 55,6; 11,2 e 0,8\% receberam $0,1,2$ ou 3 tratamentos durante 120 dias, apresentando peso semelhante após este período.

O objetivo do presente trabalho foi avaliar o ganho de peso em cordeiros, por ser a categoria mais susceptível à agressão parasitária, utilizando a avaliação seletiva com o método Famacha e o tratamento preventivo.

\section{MATERIAL E MÉTODOS}

Local do experimento e animais. O experimento foi conduzido em uma propriedade localizada no município de São Martinho da Serra, RS. O município está situado na depressão central do estado, com uma latitude $29^{\circ} 32^{\prime} 16^{\prime \prime}$ Sul e a uma longitude 5351'17" Oeste, estando a uma altitude de 453 metros acima do nível do mar. Foram selecionados 34 animais, fêmeas da raça lle de France com quatro meses de idade e acompanhados até os oito meses de idade, que compuseram dois grupos de 17 animais ( $\mathrm{G} 1$ e G2). Os animais foram locados em área de 10 ha de pastagem composta de Andropogon spp., Desmodium incomun e Paspalum notatum e eram levados para áreas de 0,9ha compostas de Cynodon spp, Brachiaria decumbens, Pennisetum glaucum, onde permaneciam durante o dia, retornando após as 17:00h. Os animais permaneceram sob manejo semelhante entre 14 de novembro de 2004 a 14 de março de 2005.

Controle parasitário seletivo. Os animais do grupo G1 foram avaliados na sua condição clínica individualmente em intervalos de 14 dias. Para tais avaliações foi utilizado o método Famacha, o escore de diarréia e o escore de condição corporal. Os animais foram tratados com moxidectina (Cydectin NF 1\%, Fort Dodge Saúde Animal) quando apresentaram algum comprometimento em relação às observações clínicas e os graus 3, 4 e 5 correspondentes ao guia Famacha. A moxidectina foi utilizada após teste preliminar de eficácia com outros produtos químicos e por demonstrar eficácia acima de $91 \%$, 21 dias pós-tratamento.

Controle parasitário preventivo. Os animais do grupo $\mathrm{G} 2$ foram tratados com o mesmo princípio ativo do grupo $\mathrm{G} 1$, sendo o mesmo utilizado em intervalos fixos de 30 dias até o final das avaliações, conforme a rotina anterior do proprietário. Estes animais foram avaliados para os mesmos parâmetros clínicos.

Exames laboratoriais e avaliação zootécnica. Amostras de fezes de todos os animais foram coIhidas diretamente da ampola retal em intervalos mensais para realização de exame da contagem de ovos (OPG) e coprocultura. A OPG foi realizada segundo a técnica de GORDON e WHITLOCK (1939) modificada e a identificação das larvas a partir da técnica de UENO e GONÇALVES (1998). Amostras da pastagem foram processadas segundo a técnica de MOLENTO (2001) modificada para determinação do grau de infestação de larvas por quilograma de massa seca (larvas/Kg/MS). Foi realizada a pesagem 
e a avaliação da condição corporal dos animais em intervalos mensais para ambos os grupos.

Analise estatística. Foi determinada a correlação dos dados pelo método de Pearson e a comparação entre o desempenho dos grupos utilizando Student t test, sendo considerado $\mathrm{P}<0,05$ para diferenças estatísticas.

\section{RESULTADOS E DISCUSSÃO}

A freqüência de tratamentos para G1 utilizando o método Famacha está demonstrada na FIGURA 1.
Houve semelhança na avaliação clínica entre G1 e G2 para a incidência de anemia dos animais com o método Famacha $(P=0,245)$. No $G 1$, somente dois animais necessitaram receber tratamento três vezes, um animal foi tratado duas vezes e três animais receberam uma dose de medicamento, sendo que $92,2 \%$ dos animais obtiveram avaliação com grau 1 e 2 durante todo o período experimental. A maior incidência de animais anêmicos foi registrada durante as avaliações no início do verão, intervalos 2 e 3 (FIGURA1). Os dados do percentual de incidência e número de tratamentos por período estão de acordo com MOLENTO et al. (2004).

\section{FIGURA 1 - PERCENTUAL DE AVALIAÇÕES DO GRAU FAMACHA(1 A 5) DO GRUPO G1 EM CORDEIROS NO PERÍODO DE NOVEMBRO DE 2004 A MARÇO DE 2005. SÃO MARTINHO DA SENA, RS $(\mathrm{N}=24)$}

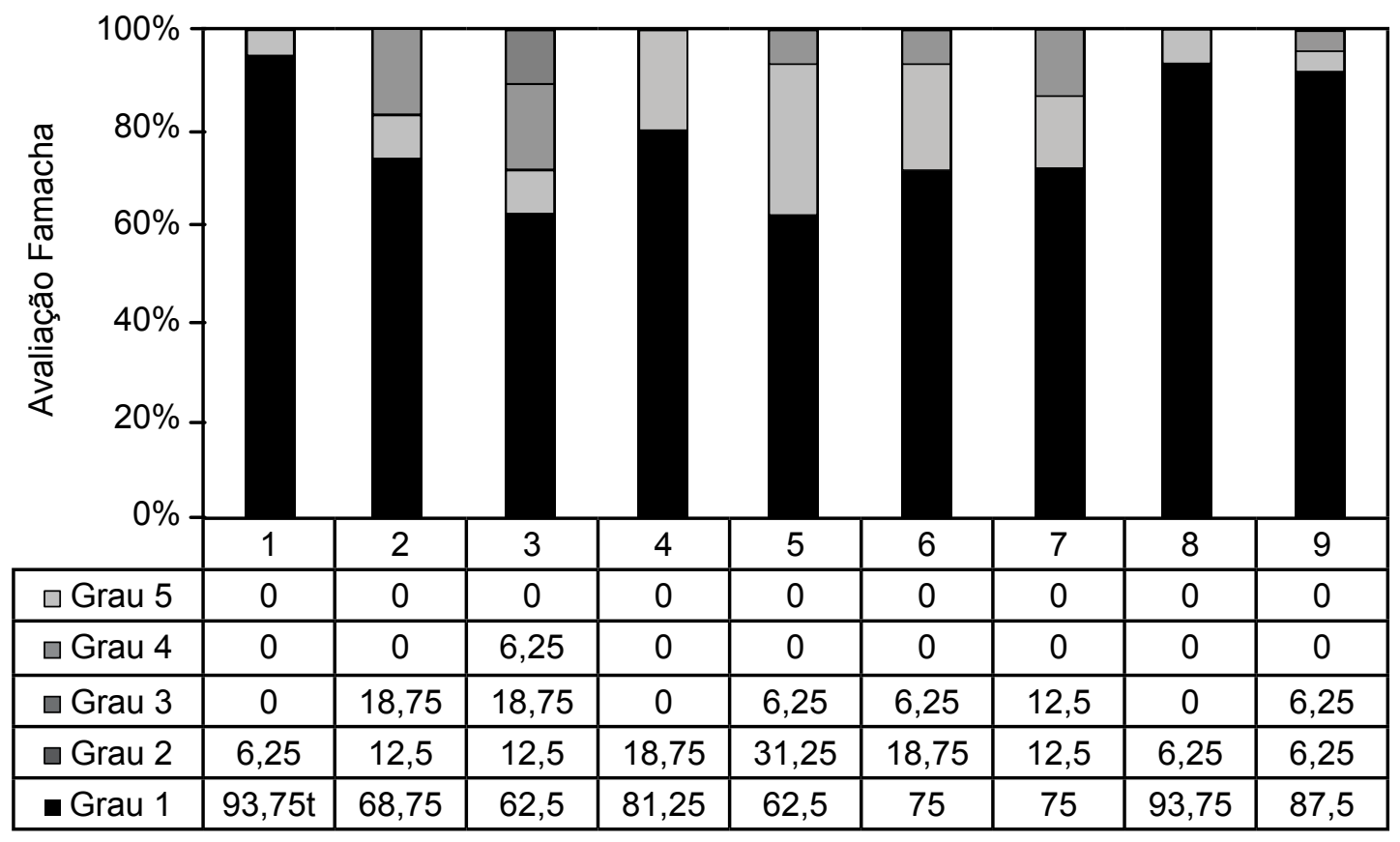

O elevado índice de animais com grau 1 e 2 $(95,8 \%)$ no grupo $G 2$ não apresentou correlação com a variável OPG no mesmo grupo. Foi determinada OPG média de 3.798 ( $\mathrm{DP}+/-2.584$ ) para esta categoria animal, sugerindo que a longa persistência do produto utilizado permitiu que os animais permanecessem sem manifestar alterações clínicas e com uma baixa taxa de estabelecimento de parasitas adultos. Entretanto, $4,2 \%$ dos animais apresentaram grau $3 \mathrm{com}$ média de OPG de 5.200 (DP+/-5280). $O$ fato de terem sido observadas altas contagens da OPG em todos os valores Famacha no dia 30 para G2 pode ser devido ao término do período de supressão da ovipostura nas fêmeas do parasita atribuído ao produto utilizado (TYRREL et al. 2002) e ao conseqüente fenômeno de equilíbrio compensatório da população parasitária decorrente do potencial biótico dos helmintos presentes. A eficácia da moxidectina foi de $57,5 \%$ após 30 dias, indicando a presença de resistência dos helmintos para esta família e sugerindo a existência de um período curto de proteção, prejudicando os benefícios do composto de longa ação, tais como, redução da contagem de ovos, contaminação da pastagem e baixo desafio parasitário.

Foram selecionados seis animais que apresentaram valores Famacha somente de grau 1 e seis animais com valores 2, 3 e 4 para correlação com os valores da OPG. A correlação Famacha e OPG em animais com valores de somente 1 foi nula, 
Desempenho de cordeiros naturalmente infectados com nematodas gastrintestinais utilizando o tratamento seletivo com o método famacha e o tratamento preventivo

entretanto a correlação foi de 0,903 para animais considerados mais susceptíveis. Este alto valor de correlação Famacha vs. OPG permite inferir sobre a possibilidade de seleção de animais menos tolerantes durante um período de cinco avaliações no momento de alto desafio parasitário.

Foi realizada correlação entre os dados de animais com valor Famacha somente de grau $1 \mathrm{e}$ animais com valores 2, 3 e 4 para Famacha e peso corporal em cinco avaliações. A correlação entre as variáveis Famacha e peso nos animais com valores de somente 1 foi nula. Entretanto, a correlação foi de 0,662 para animais com valores Famacha de 2, 3 e 4 . Este valor de correlação Famacha vs. peso fortalece a probabilidade de seleção de animais susceptíveis no momento de alto desafio parasitário. SILVA et al. (2007) determinaram valores de 94,1 e $89,3 \%$ de especificidade do método Famacha para caprinos das raças Saanen e mestiços Boer naturalmente contaminados com Haemonchus sp. e em baixa (5,54 UA/ha) e alta lotação (10,06 UA/ha) respectivamente. Os resultados do presente trabalho estão em concordância com MAHIEU et al. (2007), os quais determinaram que animais que necessitando de maior número de tratamentos obtiveram ganho de peso diário $15 \%$ menor do que animais não tratados. Os dados aqui apresentados ressaltam a utilização do método Famacha em programas de seleção intra-rebanho em ovinos lle de France após cinco avaliações.

Os dados de ganho de peso e escore corporal estão apresentados nas FIGURAS 2 e 3 e demonstram a ausência de efeitos negativos na produtividade com a adoção do método Famacha. Os animais do grupo G1 demonstraram ganho de peso total ligeiramente inferior do grupo $\mathrm{G} 2(2,8 \mathrm{~kg} \times 3,6 \mathrm{~kg})$ não apresentando diferença estatística $(P=0,110)$.

FIGURA 2 - MÉDIAS MENSAIS DE PESO (KG) DO GRUPO G1 SELETIVO (LINHA TRACEJADA) E G2 PREVENTIVO (LINHA SÓLIDA) NO PERÍODO DE NOVEMBRO DE 2004 A MARÇO DE

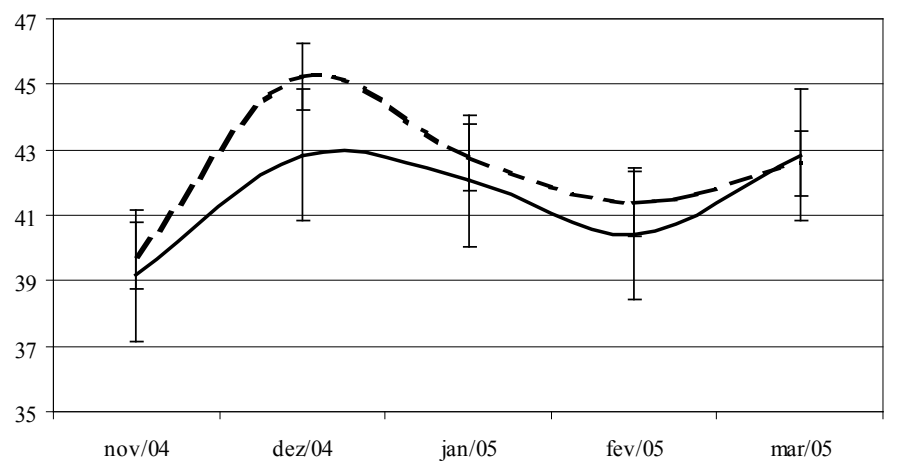

FIGURA 3 - ESCORE CORPORAL DO TRATAMENTO SELETIVO (G1) E TRATAMENTO PREVENTIVO (G2) NO PERÍODO DE NOVEMBRO DE 2004 A MARÇO DE 2005.

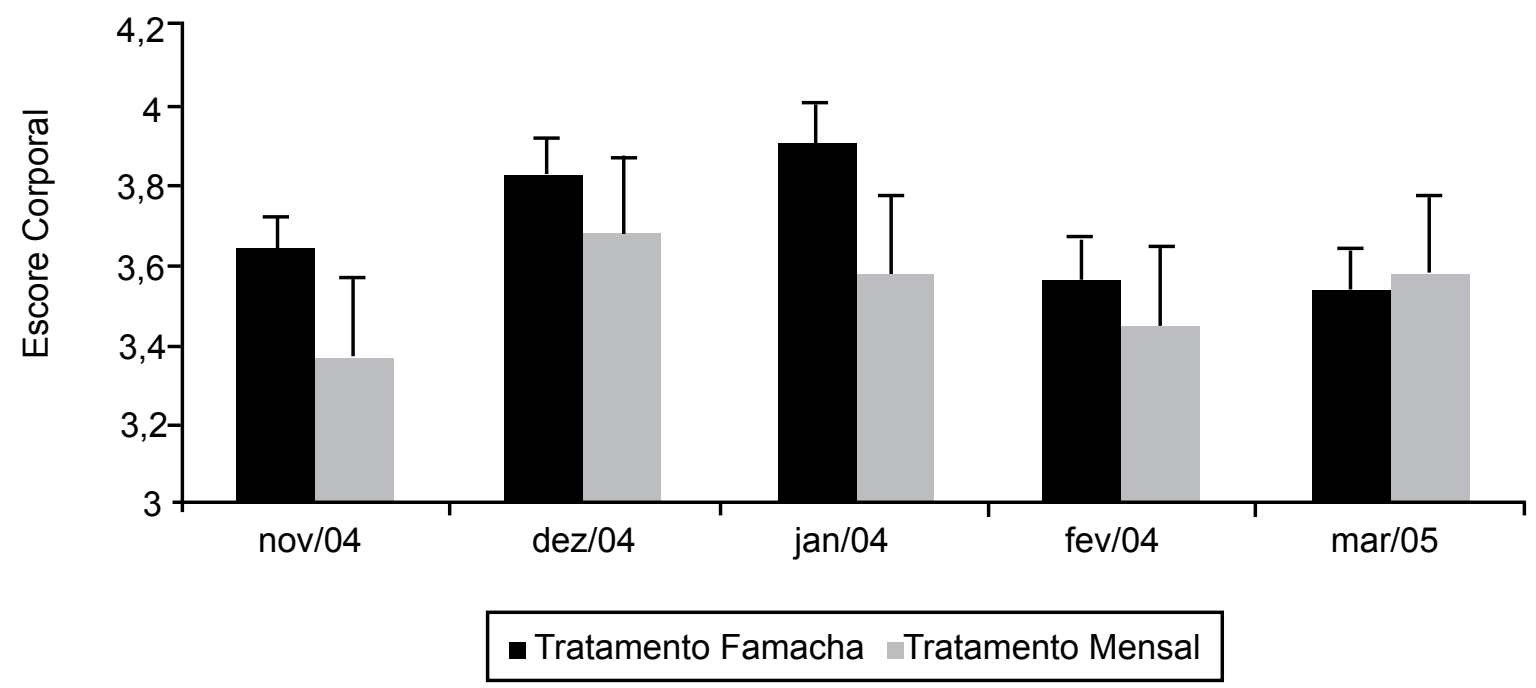


Os resultados da OPG em ambos os grupos, entre os meses de novembro a março demonstraram não haver diferença estatística (TABELA 1). A interpretação da coprocultura revelou predominância do gênero Haemonchus sp. para os tratamentos: Preventivo $(64,14 \%)$ e Seletivo $(62,94 \%)$. Foram observados os gêneros Trichostrongylus sp. $(30,94$ e
$30,46 \%)$ e Ostertagia sp. (4,0 e 3,4\%) para G1 e G2, respectivamente. O gênero Cooperia $s p$. somente foi observado no grupo Preventivo. O número de larvas de vida livre no pasto neste mesmo período aumentou consideravelmente de 776 em novembro de 2004 para 7594 em março de 2005.

TABELA 1- MÉDIA GEOMÉTRICA DA CONTAGEM DE OVOS DE HELMINTOS EM CORDEIROS POR GRAMA DE FEZES (OPG) E PERCENTUAL DOS GENNEROS ENCONTRADOS NA COPROCULTURA ENTRE O GRUPO SELETIVO (G1) E PREVENTIVO (G2) NO PERÍODO DE NOVEMBRO DE 2004 A MARÇO DE 2005.

\begin{tabular}{ccccccccccc}
\hline \multirow{2}{*}{ Gênero/Data/Grupo } & \multicolumn{2}{c}{$14 / 11 / 2004$} & $14 / 12 / 2004$ & $14 / 1 / 2005$ & \multicolumn{2}{c}{$14 / 2 / 2005$} & \multicolumn{2}{c}{$14 / 3 / 2005$} \\
\cline { 2 - 11 } & G1 & G2 & G1 & G2 & G1 & G2 & G1 & G2 & G1 & G2 \\
\hline OPG & 2110,5 & 1264,3 & 7695 & 7658 & 2447,4 & 2523,5 & 2000 & 4670,6 & 1152,6 & 2411,8 \\
\hline Haemonchus sp. & 60,5 & 47 & 70,6 & 67 & 60 & 52,4 & 60 & 54,3 & 63,6 & 100 \\
Trichostrongylus sp. & 34,5 & 45 & 23,5 & 33 & 24 & 31 & 40 & 45,7 & 30,3 & 0 \\
Ostertagia sp. & 5 & 8 & 5,9 & 0 & 0 & 12 & 0 & 0 & 6,1 & 0 \\
Cooperia sp. & 0 & 0 & 0 & 0 & 16 & 4,6 & 0 & 0 & 0 & 0 \\
\hline
\end{tabular}

A opção de tratar os animais com o sistema Seletivo (G1) promoveu uma redução de $87,5 \%$ no custo do tratamento ( $R \$ 0,24 / a n i m a l)$, quando comparado com o método de tratamento Preventivo (G2). Considerando que o impacto das infecções parasitárias pode significar a saída de produtores da atividade pecuária, o nível de economia alcançado com o presente trabalho fortalece a adoção do SICOPA com ênfase ao método Famacha em criações ovinas e caprinas.

\section{CONCLUSÕES}

Os resultados obtidos neste trabalho permitem concluir que o ganho de peso e os valores de escore corporal não foram comprometidos quando da adoção do sistema de tratamento Seletivo, permitindo que $92,2 \%$ dos animais permanecessem sem tratamento. A relação custo-benefício observada com o sistema Seletivo de controle parasitário foi superior à encontrada no tratamento Preventivo devido à diminuição do número de doses do medicamento. Os dados apresentados demonstram a aplicabilidade do método Famacha, como uma alternativa de controle parasitário eficiente quando existe alta prevalência do parasita $H$. contortus.

\section{REFERÊNCIAS}

BATH, G.F.; VAN WYK, J.A. Using the FAMACHAC system on commercial sheep farms in South Africa. In: 5th INTERNATIONAL SHEEP VETERINARY CONGRESS, XV. 2001, Cidade do Cabo, Anais... Cidade do Cabo, Associação de Veterinários da África do Sul, 2001, p.12-20.

CHANDRAWATHANI, P.; WALLER, P.J.; ADNAN, M.; HOGLUND, J. Evolution of high-level, multiple anthelmintic resistance on a sheep farm in Malaysia. Tropical Animal Health and Production, v.35, p.17-25, 2003.

ECHEVARRIA, F.; BORBA, M.F.S.; PINHEIRO, A.C.; WALLER, P.J.; HANSEN, J.W. The prevalence of anthelmintic resistance in nematode parasites of sheep in Southern Latin America: Brazil. Veterinary Parasitology, v.62, p.199-206, 1996.

GORDON, H.M.; WHITLOCK, H.V. A new technique for counting nematode eggs in sheep faeces. Journal of the Council of Scientific Industry and Research, v.12, p.50-52, 1939. 
Desempenho de cordeiros naturalmente infectados com nematodas gastrintestinais utilizando o tratamento seletivo com o método famacha e o tratamento preventivo

KAPLAN, R.M.; BURKE, J.M.; TERRILL, T.H. Validation of the Famacha ${ }^{\odot}$ eye color chart for detecting clinical anemia in sheep and goats on farms in the southern United States. Veterinary Parasitology, v.123, p.105-120, 2004.

KAWANO, E.L.; YAMAMURA, M.H.; RIBEIRO; E.L.A. Efeitos do tratamento com anti-helmíntico em cordeiros naturalmente infectados com helmintos gastrintestinais sobre os parâmetros hematológicos, ganho de peso e qualidade da carcaça. Arquivos da Faculdade de Veterinária da UFRGS, v.29, p.113-121, 2001.

MACIEL, S.; GIMENEZ, A.N.; GAONA, C.; WALLER, P.J.; HANSEN, J.W. The prevalence of anthelmintic resistance in nematode parasites of sheep in Southern Latin America: Paraguay. Veterinary Parasitology, v.62, p.207-212, 1996.

MAHIEU, M.; ARQUET, R.; KANDASSAMY, T.; MANDONNET, N.; HOSTE, H. Evaluation of target drenching using Famacha method in Creole goat: reduction of anthelmintic use, and effects on kid production and pasture contamination. Veterinary Parasitology, v.146, p.135-147, 2007.

MOLENTO, M.B. Técnica de contagem de larvas no pasto como ferramenta para diagnóstico parasitológico. In: SIMPÓSIO DAREDE DE HELMINTOLOGIAPARA AAMÉRICALATINAE CARIBE, II, 2001, Buenos Aires. Anais... FAO/ONU, Buenos Aires, 2001. CDRom.

MOLENTO, M.B. Resistência de helmintos em ovinos e caprinos. In: CONGRESSO BRASILEIRO DE PARASITOLOGIA VETERINÁRIA, XIII, Ouro Preto, Anais... CBPV, Ouro Preto, 2004. p. 32-37.

MOLENTO, M. B.; ALMEIDA, L. T. Práticas de controle parasitário adotadas por criadores de ovinos e caprinos. In: CONGRESSO BRASILEIRO DE PARASITOLOGIA VETERINÁRIA, XIII, Ouro Preto, Anais... CBPV, Ouro Preto, 2004. p. 241.

MOLENTO, M.B.; TASCA, C.; GALLO, A.; FERREIRA, M.; BONONI, R.; STECCA, E. Método Famacha como parâmetro clínico individual de infecção por Haemonchus contortus em pequenos ruminantes. Ciência Rural, v.34, p.1139-1145, 2004.

MOLENTO, M.B.; PRICHARD, R.K. Nematode control and the possible development of anthelmintic resistance. Revista Brasileira de Parasitologia Veterinária, v.8, p.75-86, 1999.
PRICHARD, R. Anthelmintic resistance. Veterinary Parasitology, v.54, p.259-268, 1994.

SILVA, H.M.; TEIXEIRA, I.A.; RESENDE, K.T.; MOLENTO, M.B.; FERREIRA, D.F.; SILVA, H.G.O.; AKINAGA, L.; FARIA, H.G. Especificidade do método Famacha $^{\odot}$ para avaliação de anemia causada por Haemonchus contortus em caprinos em Jaboticabal, São Paulo. In: CONGRESSO BRASILEIRO DE BUIATRIA, VII, Curitiba, Anais... CBB, Curitiba, 2007.

TYRREL, K.L; DOBSON, R.J.; STEIN, P.A.; WALKEN-BROWN, S.W. The effects of ivermectin and moxidectin on egg viability and larval development of ivermectin-resistant Haemonchus contortus, Veterinary Parasitology, v.107, p.85-93, 2002.

UENO, H.; GONÇALVES, P.C. Manual para o diagnóstico das helmintoses de ruminantes. Salvador: Press Color, 1998. p. 143.

VAN WYK, J.A.; STENSON, M.O.; VAN DER MEERWE, J.; VORSTER, R.J.; VILJOEN, P. Anthelmintic resistance in South Africa: surveys indicate an extremely serious situation in sheep and goat farming. Onderstepoort Journal of Veterinary Research, v.66, p.273-284, 1999.

VAN WYK, J.A.; MALAN; F.S.; BATH, G.F. Rampant anthelmintic resistance in sheep in South Africa: What are the options? In: VAN WYK, J.A.; VAN SCHALKWYK, P.C., 1997. Managing Anthelmintic Resistance in Endoparasites. Workshop/WAAVP, Cidade do Sol, África do Sul, p.51-63, 1997.

Recebido para publicação:

$06 / 06 / 2007$

Aprovado:

$05 / 12 / 2007$ 